\title{
Towards Efficient Viscous Modeling Based on Cartesian Methods for Automated Flow Simulation
}

\author{
Patrick $\mathrm{Hu}^{1}$, Hongwu Zhao ${ }^{2}$, Ramji Kamakoti ${ }^{3}$, Nagendra Dittakavi ${ }^{3}$, \\ Liping Xue ${ }^{3}$, Kan $\mathrm{Ni}^{3}$ and Shaolin $\mathrm{MaO}^{3}$ \\ Advanced Dynamics Inc., Lexington, KY 40511 \\ David D. Marshall ${ }^{4}$ \\ Aerospace Engineering Department, California Polytechnic State University \\ San Luis Obispo, California 93407-0352 \\ Michael Aftosmis ${ }^{3}$ \\ NASA Advanced Supercomputing Division, Code INA, NASA Ames Research Center \\ Moffett Field, CA 94035-1000
}

\begin{abstract}
The advanced Computational Fluid Dynamics (CFD) techniques that address the current limitations of Cartesian-based Navier-Stokes CFD schemes are explored in current investigation. Three promising methods of implementing improved wall boundary conditions are applied: (1) the enhanced diamond path stencil approach, (2) the reformulated extended extrapolation boundary condition, and (3) the ghost cell method. Several initial testing cases have been conducted with all these three boundary conditions, including the flow past a circular cylinder, flow past a flat plate at different inclined angles and flow past an AGARD RAE2822 airfoil. All the results show the effectiveness of these boundary conditions in resolving both laminar and turbulent boundary layer. Among all these methods, the extended extrapolation boundary condition attains the better results than the other two methods.
\end{abstract}

\section{Introduction}

In traditional CFD simulations with the body fitted mesh, the mesh generation is a heavy burden and sometime is very difficult, especially for complex geometries. Beside that, the body fitted mesh is proven to be difficult in dealing with very sharp corners and singular points. Therefore, Cartesian mesh has gained great popularity recently because of the short grid generation time required for complex three dimensional configurations. The advantages of the Cartesian mesh over the body fitted mesh are obvious in automated flow simulation and adaptive mesh refinement. However, the Cartesian mesh encounters the problems in the viscous wall boundary treatment. Coirier and Powell [1] demonstrated that extreme oscillations exist near the cut cells due to the non-positivity of the stencils used in viscous flux reconstruction technique. A modified diamond path stencil method was used by Delanaye and Aftosmis [2] in viscous flux reconstruction. Effectiveness of the method has been demonstrated with the Cartesian mesh outside of the boundary layer and a body fitted mesh inside of the boundary layer. However, it is still not clear how this method works in resolving both laminar and turbulent boundary layer. In addition to the non-smoothness problems, the cut cells generated by the solid surface require very small time step because of the smaller area of these cells. The problem of arbitrarily small cut cells was addressed by Clarke et al [3] as well as by Gaffney and Hassan [4]. The technique used by these researchers is the cell merging technique. To elude the time restriction problem by the cut cell, a new viscous wall treatment method was developed by Marshall and Ruffin $[5,6]$. They used the extended extrapolation method to

\footnotetext{
${ }^{1}$ Principal Scientist

${ }^{2}$ Sr. Research Scientist, corresponding author, hongwu.zhao@gmail.com

${ }^{3}$ Sr. Research Scientist

${ }^{4}$ Associate Professor
} 
compute the values at the cut cell centers directly. In this way, the time step is only determined by the regular non-cut cell size. Another boundary treatment approach was developed by Dadone et al [7] with the ghost cell method. In this method, the ghost cell values are computed by interpolation so that the viscous wall boundary condition can be satisfied. Even though the extended extrapolation method and ghost cell method can eliminate the time step restriction by the cut cell, these two methods rely more on the accuracy of the interpolation method.

In the current investigation, we studied the issue of viscous modeling with Cartesian mesh based on three viscous wall boundary treatment, including the enhanced diamond path stencil method, reformulated extended extrapolation boundary condition and ghost cell method. The test cases used to validate these methods are as follows: (1) flow past a circular cylinder; (2) flow past a horizontal flat plate; (3) flow past a flat plate inclined 30 degree angle to horizontal x-axis; and (4) flow past AGARD RAE2822 airfoil. The results are compared to identify the effectiveness of each method in resolving both laminar and turbulent boundary layer. In next section, the viscous wall boundary treatment theory will be given. This will be followed by the numerical algorithm and simulation results. The conclusion will be given in last section.

\section{Theory of Boundary Treatment}

In current investigation, three wall treatments based on Cartesian methods for viscous modeling have been studied: (1) enhanced diamond path stencil method, (2) reformulated extended extrapolation boundary condition, and (3) ghost cell method. The theory of these methods will be introduced below.

\subsection{Enhanced Diamond Path Stencil Approach}

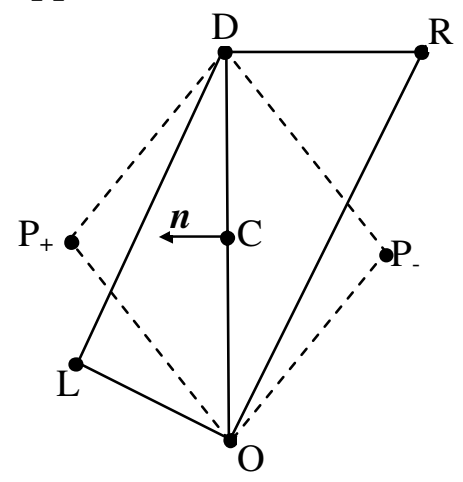

Figure 1. Schematic of standard and modified diamond control volume, standard diamond $\mathrm{CV}$ is shown by solid line and modified diamond $\mathrm{CV}$ is shown by dotted line.

The diamond path stencil technique of Delanaye et al. [2] was originally proposed and implemented for a hybrid mesh in two dimensions. We propose to apply this technique at the boundary to the viscous cells that are cut by the solid surface, thereby eliminating the body-oriented boundary layer mesh. A careful analysis of the positivity of the viscous flux stencil will be required because Delanaye et al. reported that instabilities were still present when the interface between the body oriented grid and the Cartesian grid was in the boundary layer region. Instead of using cell centroids as two of the diamond stencil points, we will select points so as to create a more regularly shaped diamond stencil. A modified diamond stencil is constructed by connecting the two vertices belonging to the edge of the Cartesian mesh with two equidistant point placed on either side of the edge thereby producing a regular-shape diamond as opposed to a distorted diamond produced by the standard diamond control volume. A schematic of the standard and modified diamond control volumes are shown in Figure 1, where the coordinates of the point $\mathrm{P}_{+}$and $\mathrm{P}$. are given by:

$$
\mathbf{r}_{P_{ \pm}}=\frac{\mathbf{r}_{O}+\mathbf{r}_{D}}{2} \pm d * \mathbf{n}
$$


where $\mathbf{r}_{\mathrm{O}}$ and $\mathbf{r}_{\mathrm{D}}$ are the position vectors of the vertices of the edge and $d$ is the minimum distance between the midpoint of the edge and the centroids of the left and right neighbors.

The next step is to obtain the values at the vertices of this control volume using interpolation and then using Gauss theorem to obtain the gradient at the mid-point of the edge. As these points require state vectors to be interpolated onto them, the interpolations must be carefully implemented, in order not to degrade overall solution accuracy. For this purpose, an interpolation scheme based on the pseudoLaplacian formula is used. Assuming the vertex to be surrounded by $M$ cells, the value at vertex $v$ can be obtained using

$$
u_{v}=\frac{\sum_{i=1}^{M} w_{i} u_{i}}{\sum_{i=1}^{M} w_{i}}
$$

where $w_{i}$ 's are the weights associated with the interpolation given by

$$
\begin{aligned}
& w_{i}=1+\lambda_{x}\left(x_{i}-x_{v}\right)+\lambda_{y}\left(y_{i}-y_{v}\right) \\
& \lambda_{x}=\frac{I_{x y} R_{y}-I_{y y} R_{x}}{I_{x x} I_{y y}-I_{x y}^{2}} \\
& \lambda_{y}=\frac{I_{x y} R_{x}-I_{x x} R_{y}}{I_{x x} I_{y y}-I_{x y}^{2}} \\
& I_{x x}=\sum_{i=1}^{M}\left(x_{i}-x_{v}\right)^{2} \\
& I_{y y}=\sum_{i=1}^{M}\left(y_{i}-y_{v}\right)^{2} \\
& I_{x y}=\sum_{i=1}^{M}\left(x_{i}-x_{v}\right)\left(y_{i}-y_{v}\right) \\
& R_{x}=\sum_{i=1}^{M}\left(x_{i}-x_{v}\right) \\
& R_{y}=\sum_{i=1}^{M}\left(y_{i}-y_{v}\right)
\end{aligned}
$$

Once the values are obtained at the vertices, the Green-Gauss theorem is used to obtain the gradient at the centroid of the modified control volume, which by construction is the midpoint of the edge (point $C$ in figure). The viscous term discretization is then based on these $1^{\text {st }}$ derivatives calculated at the mid-point of each edge, leading to a linearity preserving technique on arbitrary meshes.

\subsection{Reformulated Extended Extrapolation Boundary Condition Technique}

This method is based on an extension of a linear extrapolation technique developed by Marshall and Ruffin $[5,6]$. In their method, they used a linear extrapolation technique to obtain the state of the cut cell, see Figure 2. The state at point ' $c$ ' is used to determine the state at point ' 9 ' (centroid of the cut/surface cell) using a one-dimensional interpolation relationship along the line w-c by making use of the boundary condition at the wall ' $w$ '. The state at point ' $c$ ' is constructed either from the state of the cell containing the point or by using distance weighted interpolation of the surrounding cells (1-9) explained in the previous section. Using the no-slip boundary condition at the wall and enforcing the condition that 
tangential velocity decreases linearly to zero at the wall and normal velocity quadratically decreases to zero near the wall, we have

$$
\mathbf{u}_{9}=\left[\mathbf{u}_{c}-\left(1-\frac{\boldsymbol{\delta}_{9}}{\boldsymbol{\delta}_{c}}\right)\left(\mathbf{u}_{c} \cdot n\right) n\right]\left(\frac{\boldsymbol{\delta}_{9}}{\boldsymbol{\delta}_{c}}\right)
$$

where $\delta_{9}$ and $\delta_{c}$ are the distances from point 'w' to points '9' and 'c', respectively.

The pressure at point ' 9 ' is determined using the normal momentum equation for a flat wall, which is

$$
\frac{d p}{d n}=0
$$

Which when using $1^{\text {st }}$ order finite difference approximation yields

$$
p_{9}=p_{c}
$$

Similar relationship is obtained for temperature as well, for an adiabatic wall treatment, which is

$$
T_{9}=T_{c}
$$

For an isothermal case a similar linear extrapolation procedure is used to compute the value at point ' 9 '

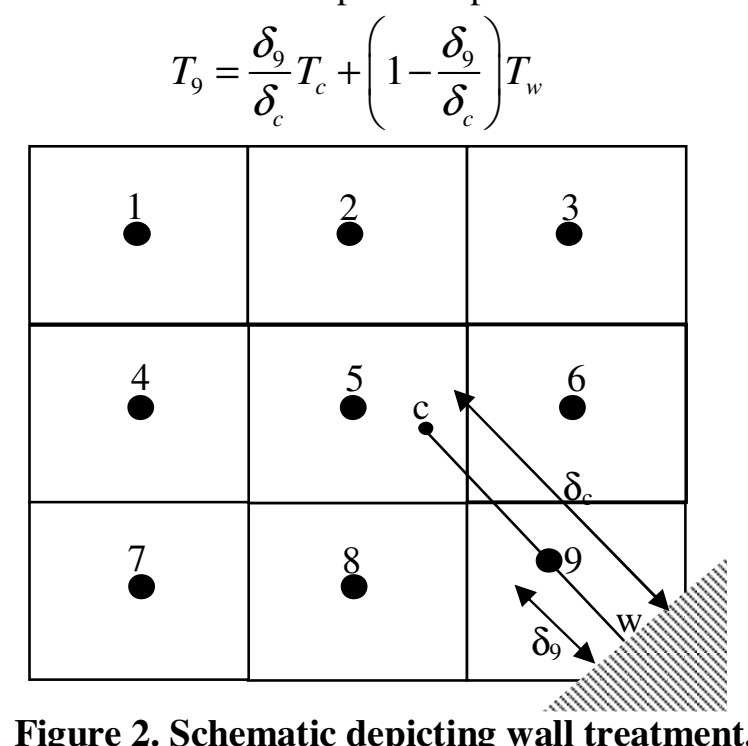

Figure 2. Schematic depicting wall treatment.

\subsection{Ghost Cell Method}

In this technique, we enforce solid wall boundary condition using the ghost cell approach, which specifies the pressure, temperature and velocity components inside the solid surface. For example, in Figure 3, the primitive variables are assigned on nodes 15 and 16 so as to obtain the correct boundary condition on the wall. The state vector of cells touching the solid wall (cells 11, 12 and 14) is found using time integration of the flow cells (cells 1-10). The points B and D are the reference points of cells 16 and 15 respectively and the values are extrapolated onto cells 16 and 15 using the states at points B, D and the wall boundary points $\mathrm{A}$ and $\mathrm{C}$. The location of the reference points $\mathrm{B}$ and $\mathrm{D}$ are determined by extending the normal from ghost cell center and body panel to a predetermined length (typically the length of the boundary cell diagonal), $\delta_{r}$. The primitive variables at the reference point are interpolated using surrounding neighbor points. A technique similar to the one used in the diamond-path stencil can be employed here as well. Once the vector state at the reference points is know, linear extrapolation is used to assign the values at the ghost cell. No-slip boundary condition for a viscous wall is enforced such that the tangential velocity is 


$$
\mathbf{v}_{T, g}=-\frac{\delta_{g}}{\delta_{r}} \mathbf{v}_{T, r e f}
$$

And the normal velocity, based on no penetration boundary condition, is given by:

$$
\mathbf{v}_{N, g}=-\frac{\delta_{g}}{\delta_{r}} \mathbf{v}_{N, r e f}
$$

The normal and tangential component of velocity is given by:

$$
\begin{aligned}
& \mathrm{v}_{N, r e f}=\left(\mathrm{v}_{r e f} \cdot \vec{n}\right) \vec{n} \\
& \mathbf{v}_{T, r e f}=\mathbf{v}_{r e f}-\mathbf{v}_{N, r e f}
\end{aligned}
$$

And $\vec{n}$ is the normal vector of the surface panel.

For pressure and temperature, we use $\frac{\partial p}{\partial n}=0$ and $\frac{\partial T}{\partial n}=0$, which leads to the following values at the ghost cell:

$$
\begin{aligned}
& p_{\text {ref }}=p_{w}=p_{g} \\
& T_{\text {ref }}=T_{w}=T_{g}
\end{aligned}
$$

where ref, w and g denote reference point, wall and ghost cell, respectively.

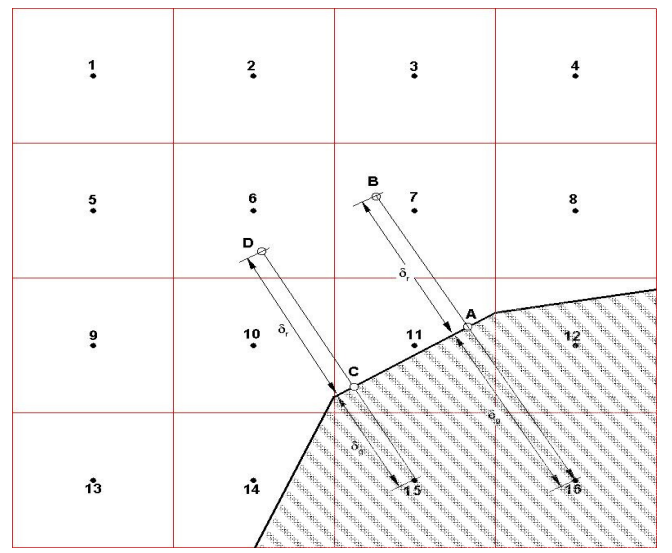

Figure 3. Schematic to demonstrate the ghost cell method.

\section{Numerical Algorithm}

To implement the different viscous wall boundary condition with Cartesian-based method, we have developed a two dimensional, compressible, finite volume code. In this code, a second- or third-order reconstruction scheme is used to compute the inviscid flux. The VANLAB limiter is used to suppress the oscillation. A second order central difference scheme is used to compute the viscous flux in the regular cells, while the three boundary treatment methods presented in Section 2 are used for the boundary cells that are cut by wall surface. A forth order Runge-Kutta method is used for time integration. MPI parallel method was also implemented in this code. The characteristics inflow and outflow boundary conditions are used to eliminate the influence of the boundary effects. Three viscous wall boundary conditions presented in the last section have been tested and the preliminary results will be discussed in following sections.

Cartesian mesh generation is very simple. In most cases, the square cells are preferred. For some boundary condition studied in this investigation, the rectangular cell may also be used, such as for the diamond path stencil method. However, for the reformulated extended extrapolation and ghost cell boundary conditions, the rectangular mesh may not work well, in particular where the aspect ratio is very high. For the diamond path stencil method, the cell cutting and merging are necessary. The detailed 
discussion for boundary cell cutting and merging procedure will be presented in the relevant test cases below.

\section{Results and Discussions}

In this section, the initial test results for all three of the viscous boundary conditions discussed in previous sections are presented. The results will be discussed and compared with a Blasius theoretical solution and/or with experimental measurement.

\section{Flow Past a Cylinder}

To validate the code, we first performed an initial test for flow past a cylinder using the Enhanced Diamond Path Stencil Approach. Figure 4(a) shows the Cartesian mesh over the cylinder, and Figure 4(b) shows the distribution of cut-cells around the cylinder wall surface. Since the boundary cells are cut by the cylinder surface with various degrees of an angle, a special treatment is needed. As shown in Figure 4(b), the outside part of the cutting cell forms a new irregular pentagon, trapezoidal, or triangle boundary cell. Because some of these cut cells are very small, they would significantly limit the time step and cause numerical instabilities during the computation. Therefore, in the present implementation, the triangle cells were merged with the outside regular non-cutting cells or with cut pentagon cells in the immediate proximity, forming a larger trapezoidal cell. Also, any trapezoidal cell with an area less than $1 / 3$ of a regular cell were merged with regular non-cutting cells that were contiguous to them. In this way, all boundary cells became trapezoidal cells. The Enhanced Diamond Path Stencil Approach was applied to all four faces of these trapezoidal cells in order to compute the velocity gradient and the viscous flux.

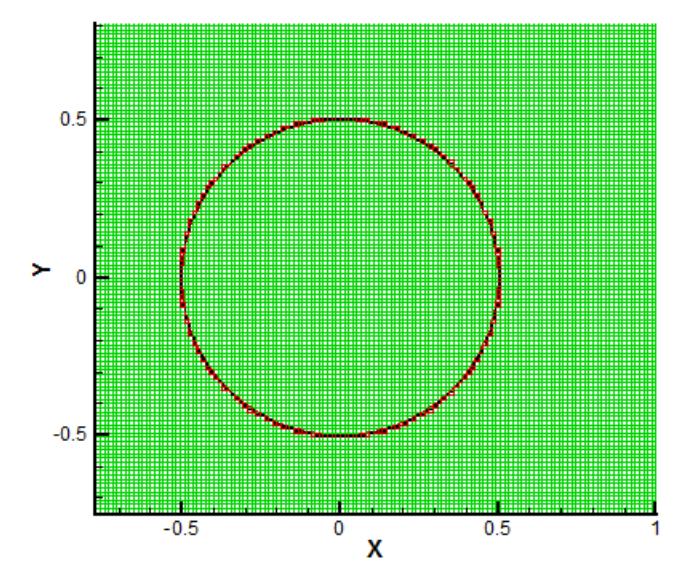

(a) Cartesian mesh and wall boundary

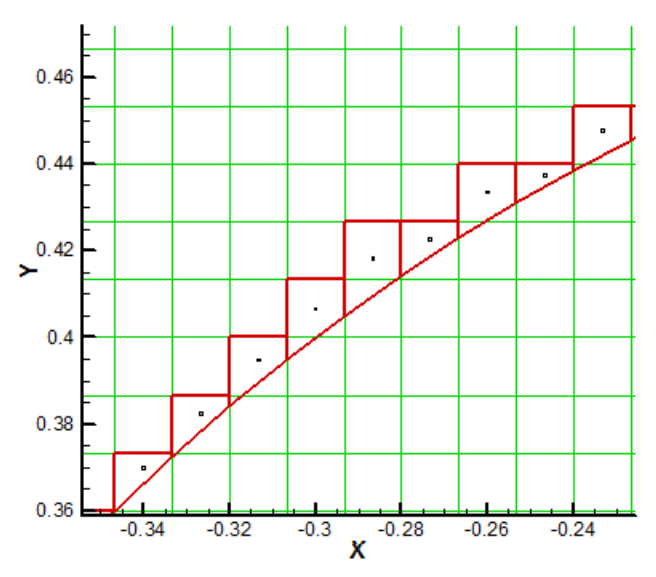

(b) Boundary cell cutting and merging Figure 4. The Cartesian mesh and boundary cell cutting and merging.

Figure 5(a) shows a subsonic flow past the circular cylinder at Mach number 0.5 and Reynolds number $1.4 \times 10^{5}$. The flow pattern and vortex shedding were captured correctly. Figure 5(b) shows the pressure distribution on the upper half of the cylinder surface. Comparison with experimental measurement [34] shows reasonable agreement. The separation location is predicted correctly. 


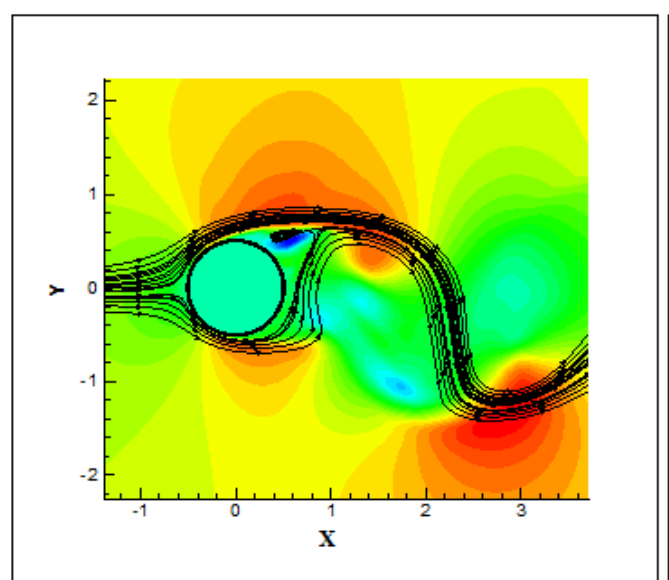

(a) Streamline and contours

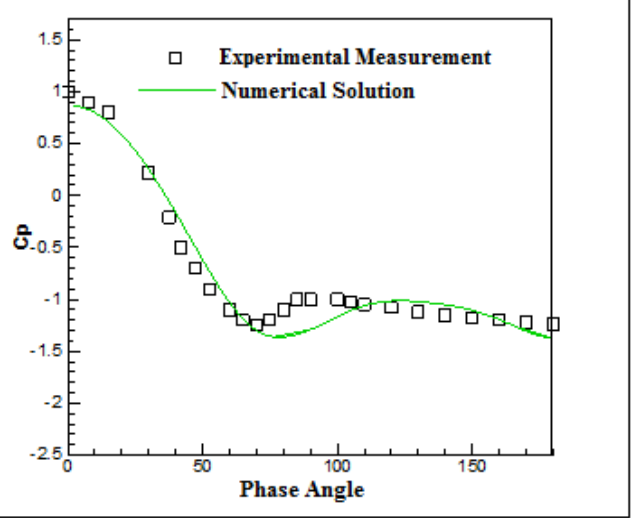

(b) Comparison of pressure on wall

Figure 5. Flow past a circular cylinder modeled by using diamond boundary condition.

\section{Flow Past a Horizontal Flat Plate}

Next, we applied all three boundary treatment techniques to the simulation of flow past a flat plate that was aligned with horizontal $\mathrm{x}$-axis. The upper and lower surfaces of the plate cut all of cells in the same proportion of cell volume. The grid was stretched in the boundary layer in order to resolve the viscous boundary layer. Figure 6(a) shows the mesh distribution. The distance from the plate's upper and lower surfaces to the center of the cell adjacent to either surface is $2 \times 10^{-5}$. The simulation was performed with Mach number 0.5 and Reynolds number $1.0 \times 10^{6}$. The two-dimensional RANS equation was solved with the Spalart-Allamars turbulence model. The flow was tripped at $\mathrm{x}=0.4$ with the trip term enforced in the Spalart-Allamars model. Figure 6(b) shows the boundary layer contour with the extended extrapolation boundary method.

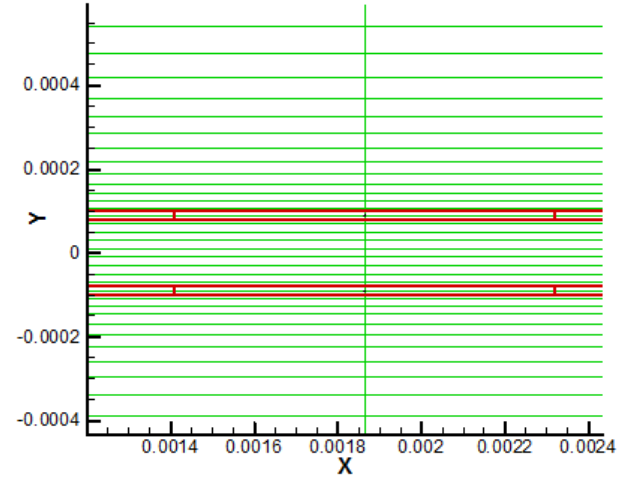

(a) Mesh distribution over a flat plate

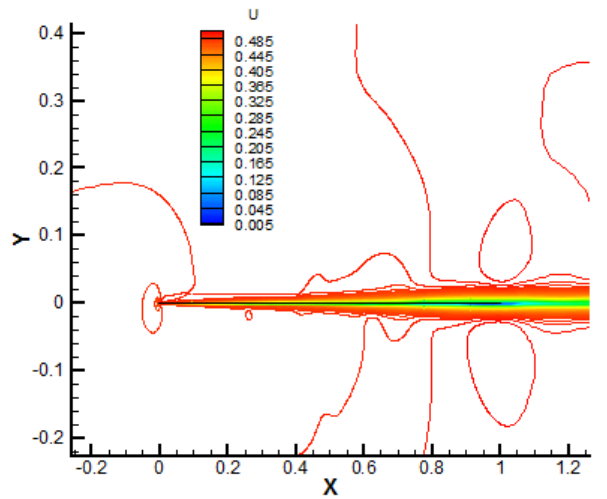

(b) Boundary layer on the flat plate Figure 6. Flow past a flat plate aligned with the horizontal $x$-axis.

The skin friction distribution along the flat plate and the turbulent boundary-layer profile at $\mathrm{x}=0.5$ are shown in Figures 7(a) and 7(b), respectively. For all three boundary treatment methods, the skin friction shown in Figure 7(a) agrees well with the Blasius laminar skin friction up to $x=0.4$. Then, due to the tripping at $\mathrm{x}=0.4$, the skin friction increases sharply before following the experimental results [51] for the turbulent boundary layer skin friction distribution. Even though all methods predict the reasonable skin friction in both laminar and turbulent regions, the extended extrapolation method gives a better agreement with the experimental measurement. Figure 7(b) indicates that while all three boundary 
treatments predict the turbulent boundary layer velocity profile [52] correctly, the extended extrapolation boundary method better resolves the log-layer.

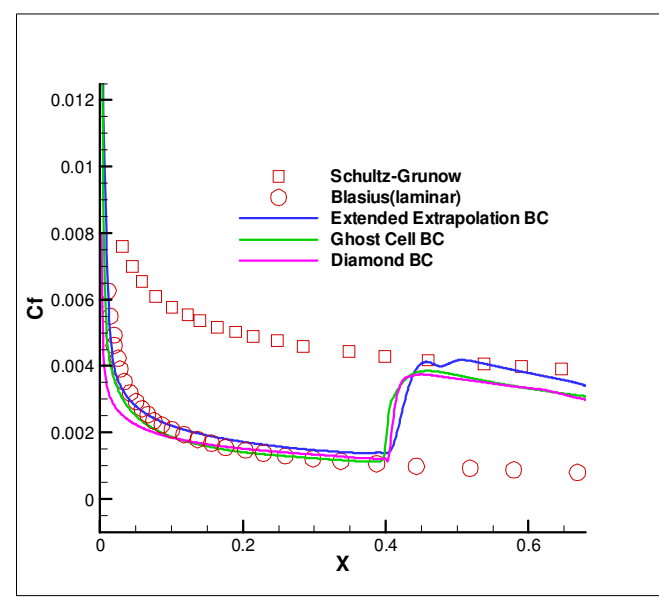

(a) Skin friction distribution

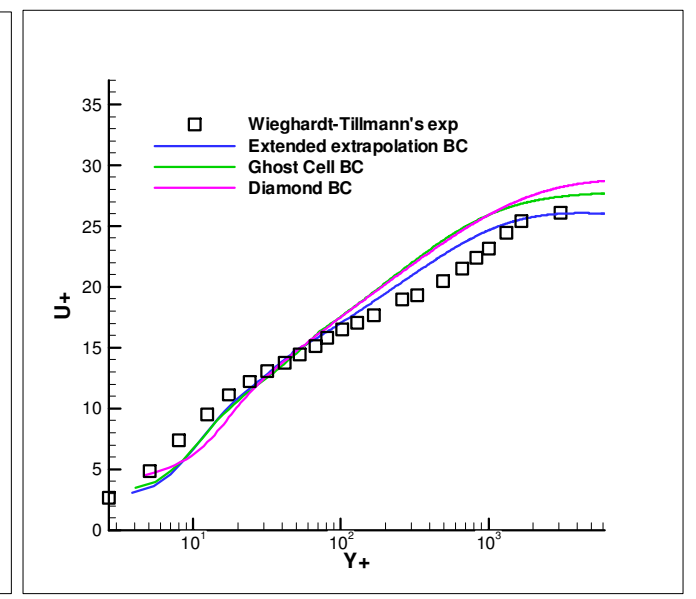

(b) Streamwise velocity profile

Figure 7. Comparison with experimental results.

\section{Flow Past a Flat Plate Inclined 30 Degrees to the Horizontal Axis}

Next, we performed the calculation for a flow past a flat plate ( 0.5 in length) inclined at a 30 degree angle to the horizontal $\mathrm{x}$-axis. In order to obtain the Blasius flow over the plate, the incoming flow passes over the plate in the direction that parallels to the plate upper and lower surfaces. The flow was simulated with all three boundary conditions. Figure 8(a) shows the Cartesian mesh around the flat plate, and Figure 8(b) shows the boundary cell cut and merge. In this case, the boundary cuts the cells in an arbitrary portion of the cell volume. For this test case, we chose the Reynolds number as $\mathrm{Re}=5000$ and the Mach number as $\mathrm{Ma}=0.5$. The laminar boundary layer was simulated and the skin friction was compared to the Blasius theoretical solution. Because a finite plate thickness must be maintained in the simulation, the influence of viscosity will cause the flow to separate at the leading edge corners. To overcome this problem, inviscid flow was simulated in the region where $\mathrm{x}<0.02$.

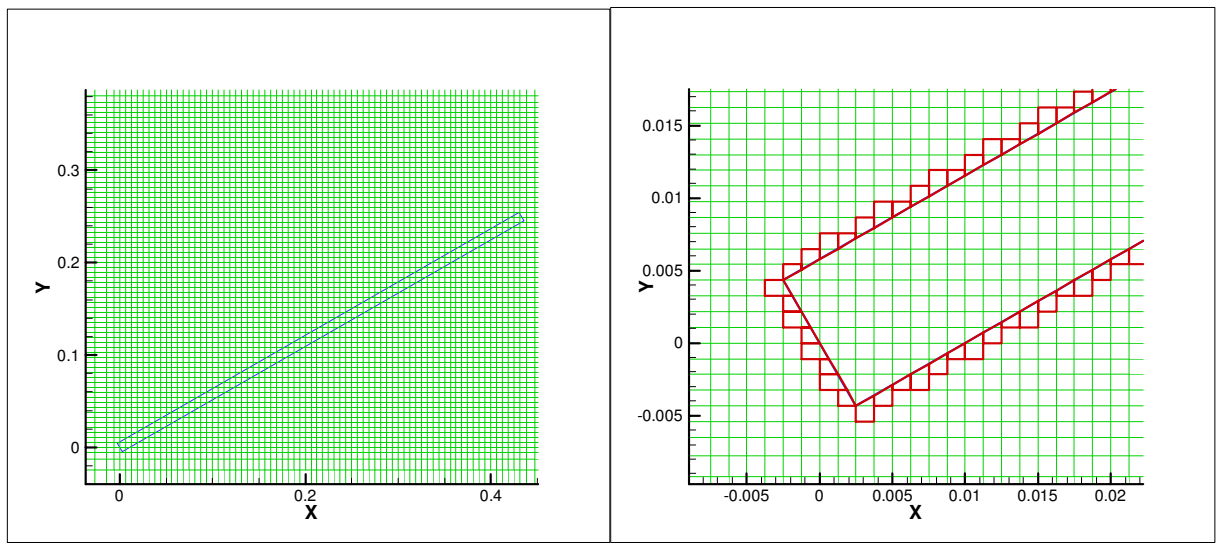

(a) Flat plate mesh

(b) Boundary cell cut and merge Figure 8. Flat plate inclined an angle of 30 degrees with horizontal axis.

Figure 9 shows the velocity contour, streamline and velocity vectors on the upper surface of the flat plate with the extended extrapolation boundary condition. It can be seen that the Blasius boundary layer was well simulated in the middle portion of the plate. Figure 10 shows the skin friction captured with all three boundary conditions on the upper surface of the plate, as well as the theoretical Blasius skin friction. For 
all three boundary treatments, the skin frictions show reasonable agreement with the Blasius theoretical solution. Figure 10(b) presents the skin frictions in log-log scale; accordingly, the linear relation is shown in the middle portion of the plate for all three boundary conditions. However, the extended extrapolation boundary condition achieves the best agreement with the theoretical solution, and with much less oscillation. The ghost cell method and the enhanced diamond method present larger oscillations, and the enhanced diamond method presents the worst skin friction among all three methods. By decreasing the mesh cell size by half, better agreements were obtained with all three methods. Nonetheless, the ghost cell and enhanced diamond methods still present some oscillations.

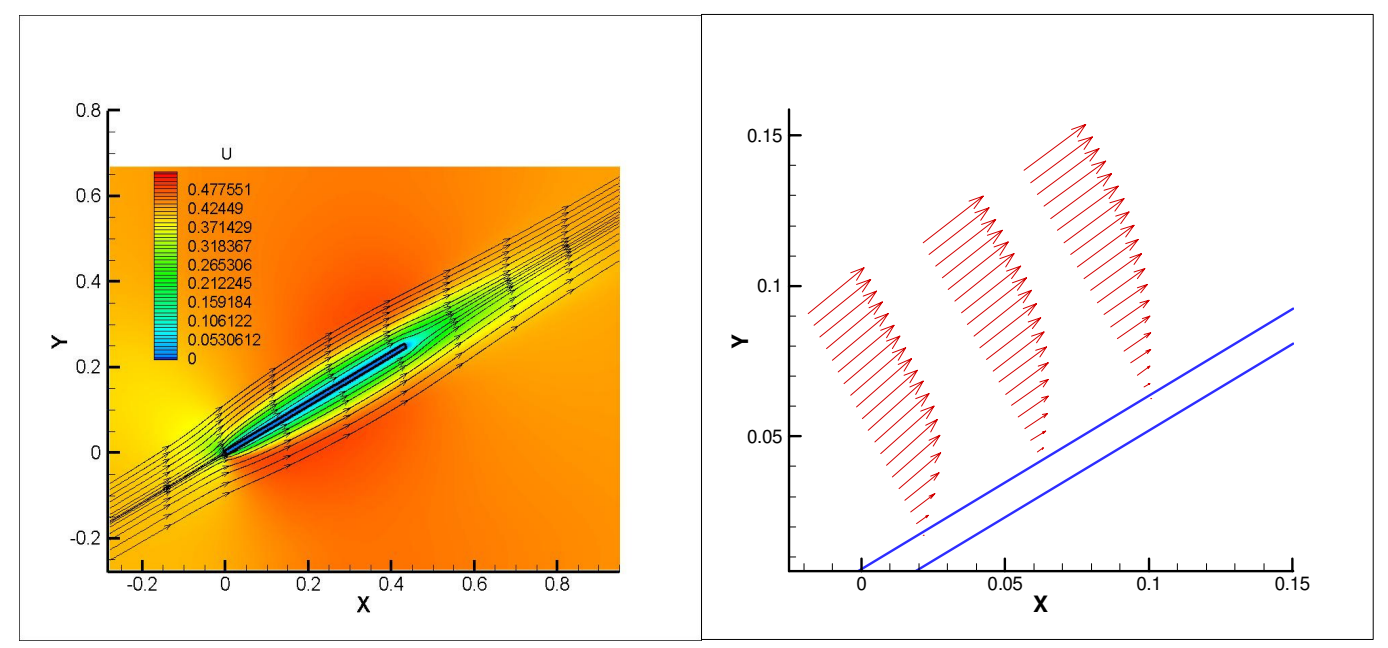

(a) Streamwise velocity contour and streamlines

(b) Velocity vectors

Figure 9. Velocity contour and vectors for a flow over a flat plate inclined an angle of $30^{\circ}$ to $x$-axis.

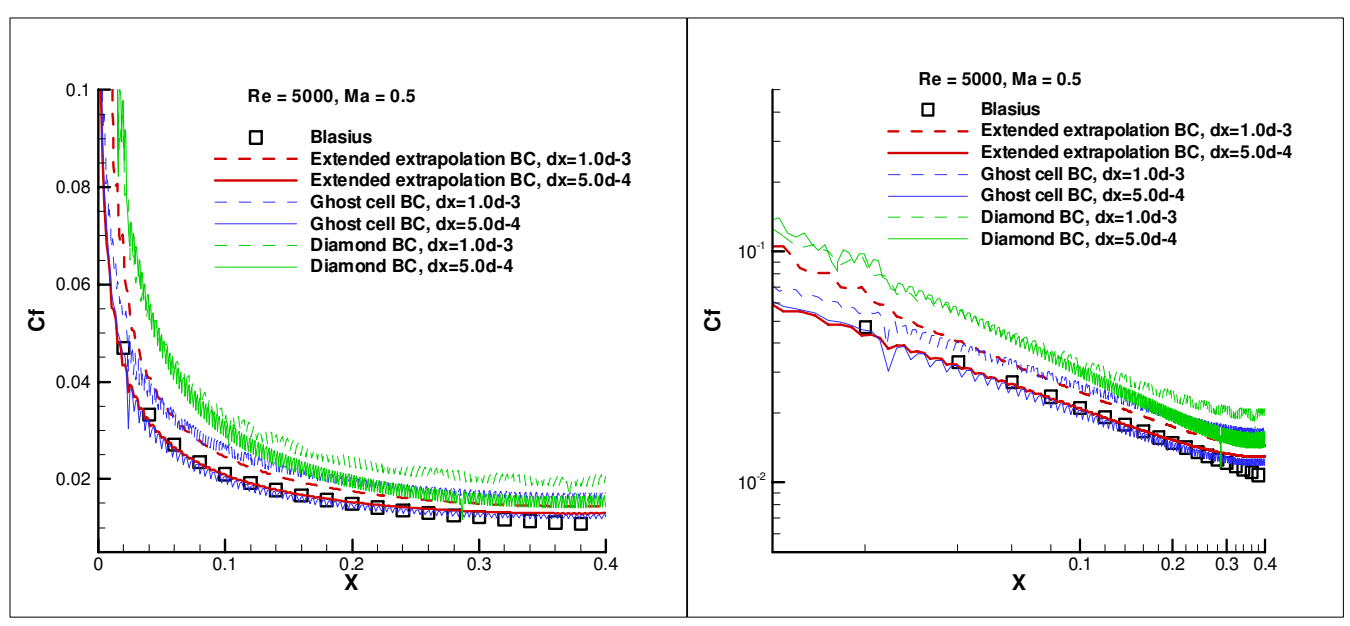

(a) Skin friction comparison

(b) Skin friction in log-log scale

Figure10. Skin friction over a flat plate inclined an angle of $30^{\circ}$ to $\mathrm{x}$-axis.

\section{Flow Past the AGARD RAE2822 Airfoil}

Next, we computed the flow past the AGARD RAE2822 airfoil. Figure 11(a) shows the Cartesian mesh around the airfoil. Figure 11(b) shows the boundary cell cutting and merging. In this simulation, we chose the Reynolds number as $\operatorname{Re}=1.0 \times 10^{5}$ and the Mach number as $\mathrm{Ma}=0.676$. The incoming flow is at an angle of attack of $1.92^{\circ}$ to $x$-axis. Figure 12 shows the velocity and pressure contours, as well as the 
streamlines in the flow field with the extended extrapolation boundary treatment. Figures 13(a) and 13(b) show the pressure coefficient captured by the extended extrapolation boundary treatment and ghost cell boundary treatments respectively on the airfoil surface. With both the extended extrapolation and ghost cell boundary treatments, the simulations achieved good agreement with the experimental measurement. By decreasing the mesh cell size, better agreement was obtained. To fully resolve the viscous flow at high Reynolds number, mesh refinement need to be used to refined the mesh along the surface interface. Cartesian mesh has great advantage in implementing adaptive mesh refinement (AMR) technology, which has made Cartesian method a powerful tool in simulating viscous flow with complex geometry configuration. The AMR method will be implemented in our future research on Cartesian method.

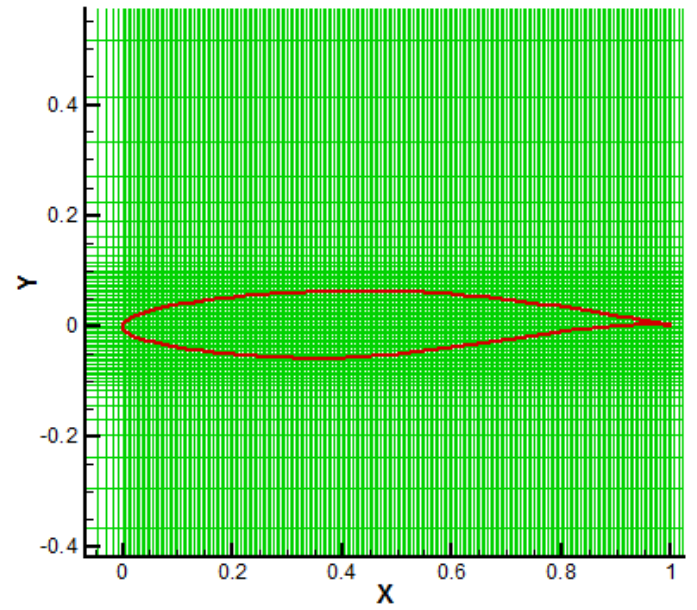

(a) RAE2822 mesh

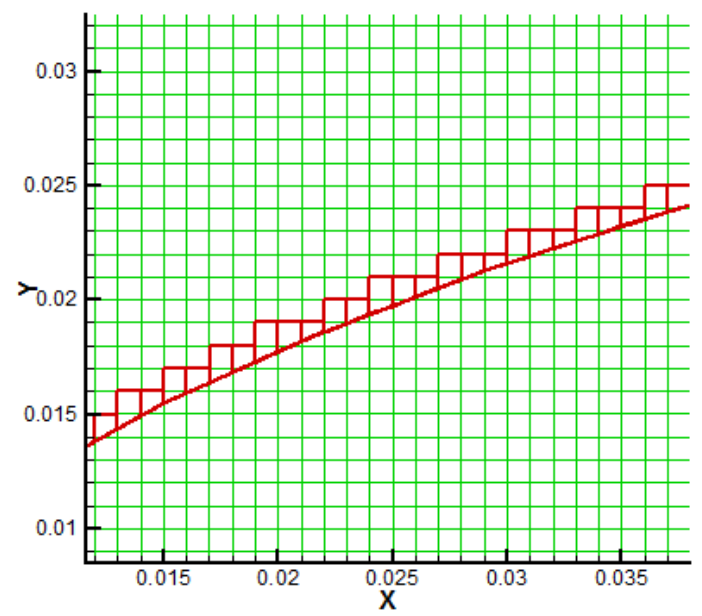

(b) Boundary cell cut and merge

Figure 11. RAE2822 mesh and boundary cell cut.

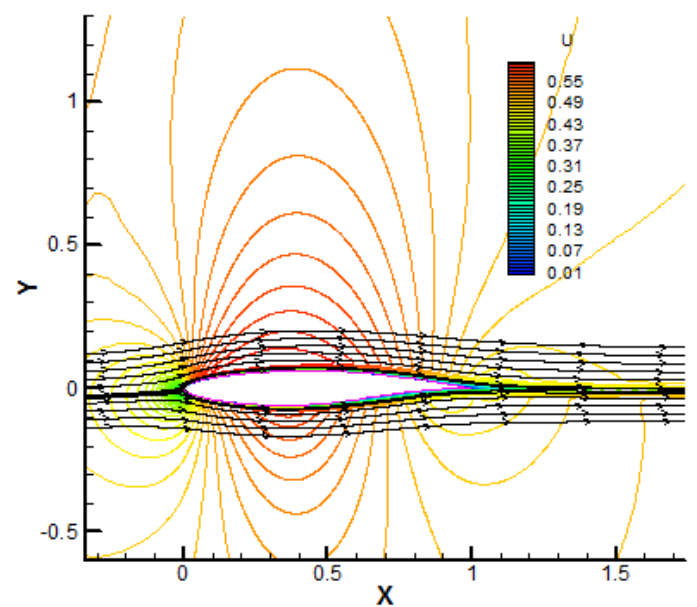

(a) Velocity contour and streamlines

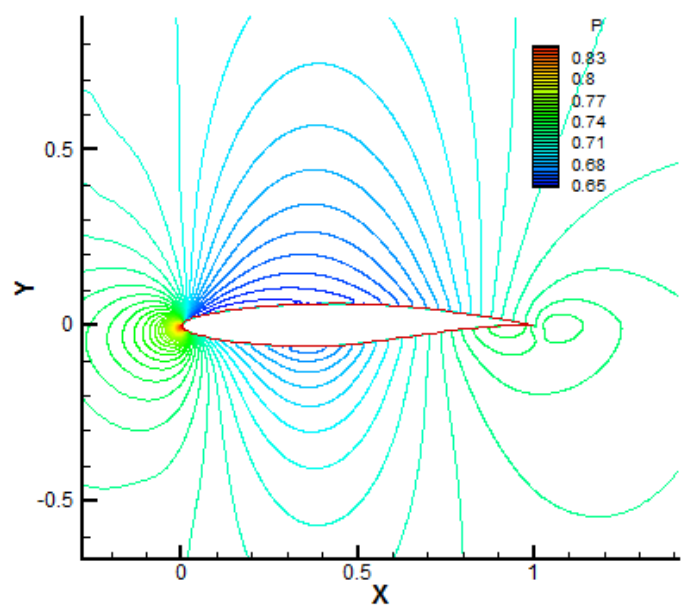

(b) Pressure contour

Figure 12. Velocity and pressure contour over RAE2822 airfoil. 


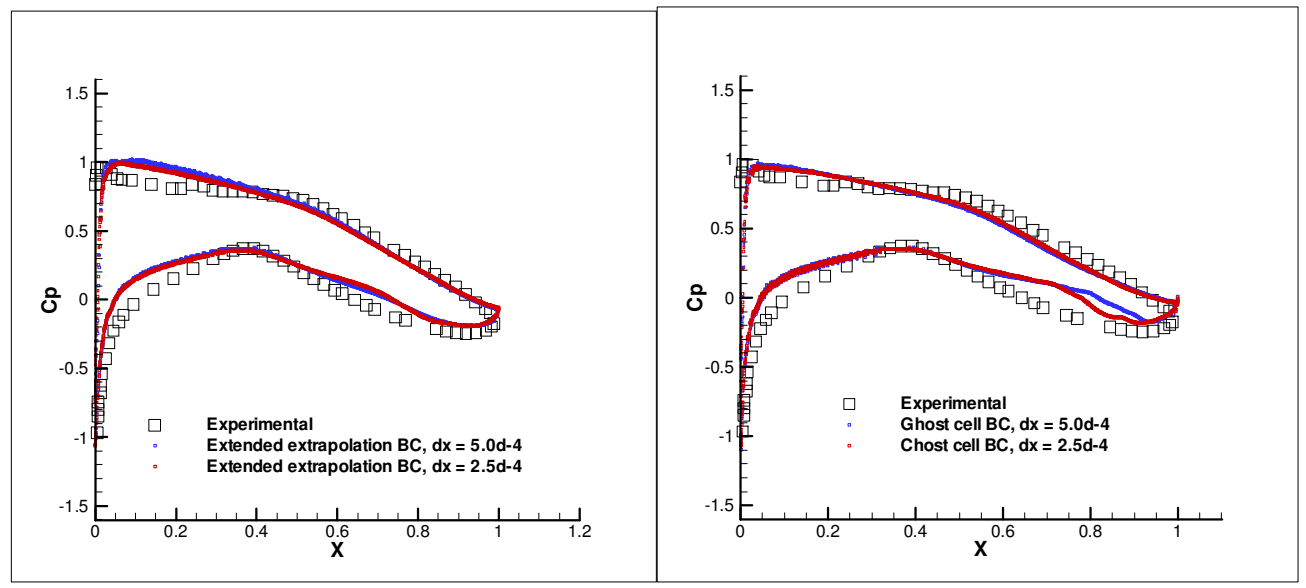

(a) Extended extrapolation BC

(b) Ghost cell BC

Figure 13. Pressure coefficient over RAE2822 airfoil.

\section{Conclusions}

In this investigation, the wall boundary treatments for viscous modeling based on Cartesian methods have been studied. Some preliminary results have been generated, and the effectiveness of the three proposed boundary treatments for resolving viscous boundary layer is demonstrated. The quantitative comparison of the present calculation with the Blasius theoretical solution (for the flat plate) and with experimental measurement (for the cylinder and the AGARD RAE2822 airfoil) demonstrates that the proposed Cartesian-based approaches is capable of resolving both laminar and turbulent boundary layers. Among all three viscous boundary treatment methods, the extended extrapolation method attains the best viscous boundary simulation, in which smaller oscillations in pressure and smoother skin frictions on the surface have been observed. Adaptive mesh refinement method will be implemented in our future research to resolve the turbulent boundary layer on the surface which intercepts the Cartesian mesh with arbitrary angles.

\section{References}

1. Coirier, W. J. and Powell, K. G., "Solution-Adaptive Cartesian Cell Approach for Viscous and Inviscid Flows," AIAA Journal, Vol. 34, No. 5, May 1996, pp. 938-945.

2. Delanaye, M, Aftosmis, M. J., Berger, M. J., Liu, Y. and Pulliam, T. H. (1999) "Automatic Hybrid-Cartesian Grid Generation for High-Reynolds Number Flows around Complex Geometries.” 37th Aerospace Sciences Meeting \& Exhibit, Reno, NV. AIAA-99-0777.

3. Clarke, D. K., Salas, M. D., and Hassan, H. A., "Euler Calculations for Multielement Airfoils sing Cartesian Grids," AIAA Journal, Vol. 24, No. 3, March 1986, pp. 353-358.

4. Gaffney, R. L., Hassan, H. A., and Salas, M. D., "Euler Calculations for Wings Using Cartesian Grids," AIAA 25th Aerospace Sciences Meeting, Reno, NV, Jan. 1987, AIAA-87-0356.

5. Marshall, D. D. (2002) "Extending the Functionalities of Cartesian Grid Solvers: Viscous Effects Modeling and MPI Parallelization," Ph.D. Thesis, Georgia Institute of Technology, Atlanta, GA.

6. Marshall, D. D. and Ruffin, S. M. (2004) "An Embedded Boundary Cartesian Grid Scheme for Viscous Flows using a New Viscous Wall Boundary Condition Treatment." 42nd Aerospace Sciences Meeting, Reno, NV, AIAA-2004-0581.

7. Dadone A., Bari P., and Grossman B., "Ghost-Cell Method for Inviscid Two-Dimensional Flows on Cartesian Grids," AIAA Journal, vol.42, no.12, 2004. pp. 2499-2507. 